DOI: 10.36868/ejmse.2020.05.03.156

\title{
SPECTRAL ANALYSIS OF OBTAINED QUANTUM CARBON DOTS FROM FOOD PRODUCTS
}

\author{
Zuzanna IWIŃSKA ${ }^{1}$, Paweł PIETRUSIEWICZ2, * \\ ${ }^{1}$ Department of Physics, Faculty of Production Engineering and Materials Technology, Czestochowa University of \\ Technology, 19 Armii Krajowej Str., 42-200 Częstochowa, Poland
}

\begin{abstract}
The article describes the preparation and testing of optical properties of carbon quantum dots. optical properties were tested using a UV-VIS spectrophotometer and spectrometer. The maximum absorbance was determined in the range of $\lambda \sim 377$ to $\sim 408 \mathrm{~nm}$. All solutions obtained showed an emission corresponding to the wavelength in the green light range. Furthermore, the effect of solution concentration on the optical properties of carbon quantum dots was investigated.
\end{abstract}

Keywords: quantum carbon dots; nanoparticles; UV-VIS absorption.

\section{Introduction}

Carbon quantum dots (CQDs) are a new class of nanomaterials obtained in 2004. Since their discovery, their popularity has been steadily increasing, which is reflected in the increasing number of publications with the word "carbon dots" in the title [1-3]. Since the first receipt of carbon quantum dots, many new synthesis methods and properties have been described, and their use has been demonstrated in many different areas. In opposition to semiconductor quantum dots, CQDs aren't toxic. The toxicity of traditional carbon quantum dots is due to the use of heavy metals in their production. Therefore, the use of QDs in clinical trials may be problematic [4-6]. Moreover, another disadvantage with semiconductor quantum dots is the costly and often polluting the environment synthesis methods. Therefore, CQDs can be an alternative for them because they can be produced in an environmentally friendly and inexpensive way [4,7]. CQDs synthesis methods are divided into two main groups: bottom-up and top-down. Bottom up methods include: microwave synthesis, thermal decomposition, hydrothermal synthesis, plasma treatment. While, top-down methods include ultrasonic synthesis, oxidizes electrochemical nannies, chemical oxidation, arcing. The first of the methods usually used to synthesize CQDs utilize an impact of energy on a carbon source. An example of such a method is laser ablation. However, the nanoparticles obtained in the above manner weren't fluorescent. These particles began to show fluorescence only after surface functionalization $[2,8]$. Replacement to the synthesis methods requiring the consumption of a large amount of energy is the method of synthesis in hydrothermal conditions. The synthesis is carried out in an aqueous solution of appropriate compounds, at high temperature, under high steam pressure. Furthermore, this method is considered simple and cost-effective due to the use of cheap apparatus and the possibility of obtaining CQD from practically any biomass. CQDs are composed of a carbogenic core and crystalline or amorphous parts. They also have functional groups on their surface [3$4,9-10]$. They are usually oxygen groups, what is the causa that CQD gain good solubility in aqueous solutions [3]. Excitation-dependent photoluminescence is another and probably most commonly used property of CQD. However, the exact fluorescence mechanism of these 
nanoparticles is not yet fully understood $[4,11]$. CQDs show absorbance in the UV region with the tail extending to the visible area [3,12-13]. These nanoparticles are used, among others in bioimaging and as biosensors. Due to, multi-color emission-dependent emission and high photostability, biosensors based on CQDs have been created for visual monitoring, among others copper, potassium iron and nucleic acids [14-15]. Moreover, chemical sensors using CQDs to detect $\mathrm{Hg}^{2+}$ mercury ions have also been developer [16].

In this paper, CQD was obtained in hydrothermal conditions from gelatin and a hypoallergenic infant formula replacement. Gelatin is a virtually colourless and tast eless watersoluble protein prepared from collagen and used in food preparation. The above food products were used due to the high content of amino acids, which may affect the quantum efficiency of the CQDs obtained [17].

\section{Experimental}

Four samples were prepared as follows: samples 12 and 3 were prepared from gelatin, while sample 4 from infant milk replacer. Sample 1 was prepared from $13.2 \mathrm{~g}$ of gelatin dissolved in $40 \mathrm{ml}$ distilled water with the addition of $2.5 \mathrm{ml}$ spirit vinegar. Sample 2 was prepared from $1 \mathrm{~g}$ of gelatin dissolved in $40 \mathrm{ml}$ distilled water with the addition of $1 \mathrm{ml}$ spirit vinegar. Sample 3 was prepared from $1 \mathrm{~g}$ of gelatin dissolved in $40 \mathrm{ml}$ distilled water and sample 4 was prepared with $4.5 \mathrm{~g}$ of hypoallergenic milk and $30 \mathrm{ml}$ of distilled water. Secondly, the solutions were placed in an autoclave - stainless steel vessel with a teflon liner - and placed in the oven. Sample 1 was heated for $6 \mathrm{~h}$ at $180^{\circ} \mathrm{C}$. Sample 2 and 3 were heated for $3 \mathrm{~h}$ at $200^{\circ} \mathrm{C}$. Sample 4 was and heated for $3 \mathrm{~h}$ at $180^{\circ} \mathrm{C}$. Finally, the vessel was cooled to room temperature. Solutions were subjected to multi-stage filtration using ultrasounds, a centrifuge and PES filters. Moreover, solutions were washed with dichloromethane to remove the unreacted organic moieties Sample 1 was diluted three times, five times and ten times.

\section{Results and discussion}

The UV-VIS cary 100 Bio spectrophotometer from Varian and the Al-Prof SM1 Hm spectrometer were used to characterize the optical properties of carbon quantum dots. UV-VIS spectra of all samples are shown in Fig. 1.

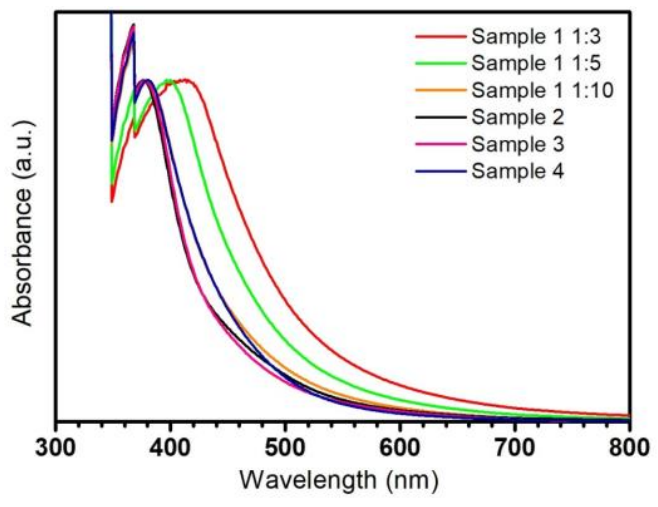

Fig. 1. UV-VIS absorption spectrum

The absorbance spectrum of sample 1 diluted three times shows a broad peak in the range of $\sim 368 \mathrm{~nm}$ to $\sim 440 \mathrm{~nm}$, with a maximum at $408 \mathrm{~nm}$. The maximum absorbance of sample 1 diluted five times is $397 \mathrm{~nm}$, the absorbance peak has a smaller range than that of the sample 
diluted three times, i.e. from $\sim 368 \mathrm{~nm}$ to $\sim 420 \mathrm{~nm}$. Sample 1 diluted ten times had an absorbance peak extending less than that of the previous two solutions, ranging from $\sim 368 \mathrm{~nm}$ to $\sim 400 \mathrm{~nm}$, with a maximum absorbance of $380 \mathrm{~nm}$. For that reason, it can be assumed that the concentration of the tested solution affects the shift of the absorption band. In the above cases, it was observed that in the case of solutions with lower concentration, the absorption band shifts in the direction of shorter waves. The absorbance spectrum of sample 2 shows a broad peak in the range of $368 \mathrm{~nm}$ to $\sim 395 \mathrm{~nm}$, with a maximum absorbance of $377 \mathrm{~nm}$. The absorbance spectrum of sample 3 shows a wide peak in the range close to sample 2, i.e. from $\sim 368 \mathrm{~nm}$ to $\sim 395 \mathrm{~nm}$, with a maximum, which, as in sample 2 , is $377 \mathrm{~nm}$. The absorbance spectrum of sample 4 shows a broad peak in the range of $\sim 368 \mathrm{~nm}$ to $\sim 400 \mathrm{~nm}$, with a maximum of $380 \mathrm{~nm}$. Based on the range of absorbance spectra and its maximum values, it can be assumed that there was an electronic transition from the n-binding orbital to the $\pi *$ anti-binding orbital in all of the above samples.

Using the Al-Prof SM1 Hm spectrometer, the maximum CQD excited emission was determined. The results are presented in Fig. 2-7.

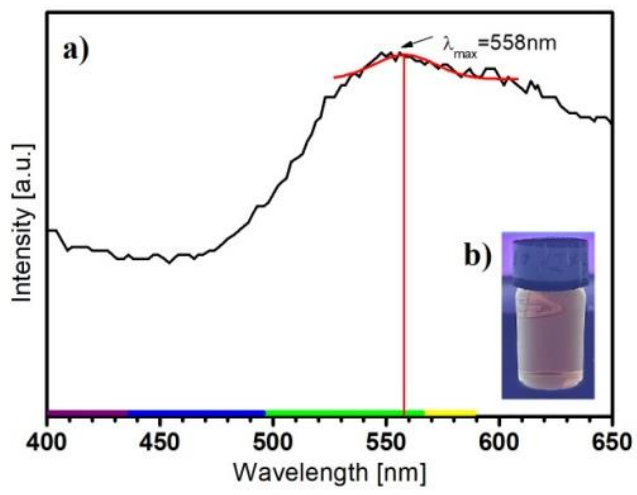

Fig. 2. Sample 1 diluted three times:

a) photoluminescence emission spectrum, b) sample under UV light

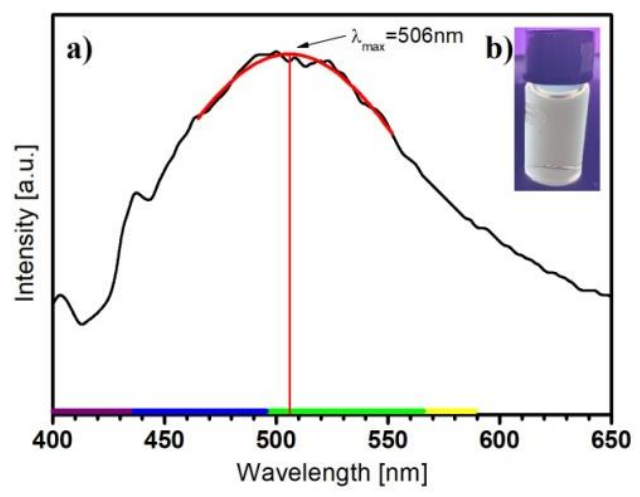

Fig. 4. Sample 1 diluted ten times:

a) photoluminescence emission spectrum, b) sample under UV light

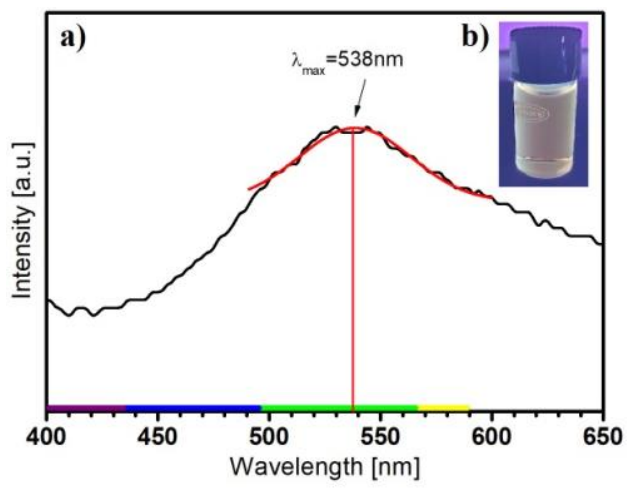

Fig. 3. Sample 1 diluted five times:

a) photoluminescence emission spectrum,b) sample under UV light

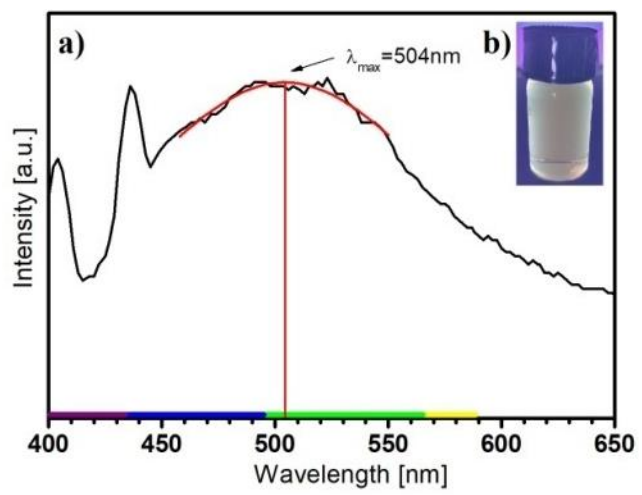

Fig. 5. Sample 2: a) photoluminescence emission spectrum, b) sample under UV light 


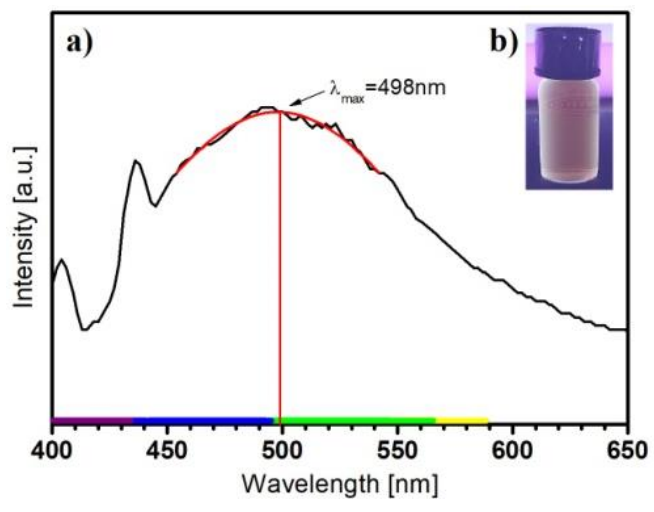

Fig. 6. Sample 3: a) photoluminescence emission spectrum, b) sample under UV light

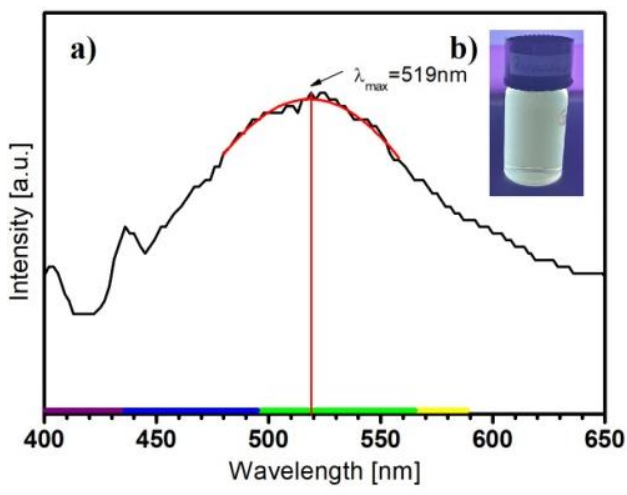

Fig. 7. Sample 4: a) photoluminescence emission spectrum, b) sample under UV light

Figures 2-7 presents the photoluminescence spectra of the solutions and their photos in ultraviolet light. The maximum emission of excited carbon quantum dots was determined from $498 \mathrm{~nm}$ to $\sim 558 \mathrm{~nm}$. Based on the above photoluminescence spectra, it can be assumed that the concentration of the solution may affect the shift of the photoluminescence spectrum. In the above cases, it was observed that the lower the concentration of the solution, the more the spectrum is shifted towards short waves. In each of the solutions obtained, the maximum emission corresponds to the wavelength characteristic of green emission.

\section{Conclusions}

Based on the performed spectrophotometric, spectrometric and literature data, it can be assumed that carbon quantum dots from gelatin and infant formula replacement were obtained. The effect of solution concentration on optical properties was also investigated. Based on the obtained UV-VIS spectra, it can be assumed that the concentration of the solution affects its optical properties. The sample with the highest concentration showed a broad peak with a maximum $\lambda_{\max }=408 \mathrm{~nm}$, while the UV - VIS spectrum of the sample with a lower concentration showed a peak in the lower wavelength range and with the maximum shifted towards ultraviolet waves, $\lambda_{\max }=380 \mathrm{~nm}$. The excitation of synthesized quantum carbon dots was induced by a UV lamp emitting light in the range from 360 to $370 \mathrm{~nm}$.

\section{References}

[1] Xu X., Ray R., Gu Y., Ploehn J. H., Gearheart L, Raker K., Scrivens A. W., Electrophoretic analysis and purification of fluorescent single-walled carbon nanotube fragments, Journal of the American Chemical Society, 2004, 126, pp. 12736-12737

[2] Jelinek R., Carbon Quantum Dots Synthesis, Properties and Applications, Springer, 2017

[3] Wang R., Lu K. Q., Tang Z. R., Xu J. Y., Recent progress in carbon quantum dots: synthesis, properties and applications in photocatalysis, Journal of Materials Chemistry A, 2017, 5, pp. 3717-3734

[4] M.J. Molaei, A review on nanostructured carbon quantum dots and their applications in biotechnology, sensors, and chemiluminescencje, Talanta, 2019, 196, pp. 456-478

[5] Lim S. Y., Shen W., Gao Z., Carbon quantum dots and their applications, Chemical Society Reviews, 2014, 44,pp. 362-381 
[6] Geys J., Nemmar A., Verbeken E., Smolders E., Ratoi M., Hoylaerts M. F., Nemery B., Hoet P. H. M., Acute Toxicity and Prothrombotic Effects of Quantum Dots: Impact of Surface Charge, Environ Health Perspect, 2008, 116, pp. 1607-1613

[7] Zuo P., Lu X., Sun Z., Guo Y., He H., A review on syntheses, properties, characterization and bioanalytical applications of fluorescent carbon dots, Microchimica Acta, 2016, 183, pp. 519-542

[8] Sun Y., Zhou B., Wang W., Fernando S., Pathak P., Meziani J. M., Haruff A. B., Wang X., Luo G. P., Yang H., Kose E. M., Chen B., Veca M., Xie S., Quantum-sized carbon dots for bright and colorful photoluminescence, Journal of the American Chemical Society, 2006, 128, pp. 7756-7757

[9] Duran N., Simões M.B., de Moraes A., Favaro W.J., Seabra A.B., Nanobiotechnology of Carbon Dots: A Review, Journal of biomedical nanotechnology, 2016, 12, pp. 1323-1347

[10] Li H., He X., Kang Z., Huang H., Liu Y., Liu J., Lian S., Tsang C.H., Yang X., Lee S.T., Water-soluble fluorescent carbon quantum dots and photocatalyst design, Angewandte Chemie - International Edition, 2010, 49, pp. 4430 - 4434

[11] Liu M., Chen B. Li Ch., Huang Ch., Carbon dots: synthesis, formation mechanism, fluorescence origin and sensing applications, GreenChemistry, 2019, 21, pp.449-471

[12] Zhou J., Booker C., Li R., Zhou X., Sham T.K., Sun X., Ding Z., An Electrochemical Avenue to Blue Luminescent Nanocrystals from Multiwalled Carbon Nanotubes (MWCNTs), Journal of the American Chemical Society, 2007, 129, pp. 744-745

[13] Wang Y., Hu A., Carbon quantum dots: synthesis, properties and applications, Journal of Materials Chemistry C, 2014, 2, pp. 6921-6939

[14] Wang J., Li R. S., Zhang H. Z., Wang N., Zhang Z., Huang C. Z., Highly fluorescent carbon dots as selective and visual probes for sensing copper ions in living cells via an electron transfer process, Biosensors and Bioelectronics, 2017, 97, pp. 157-163

[15] Li H., Zhang Y., Wang L., Tian J., Sun X., Nucleic acid detection using carbon nanoparticles as a fluorescent sensing platform, Chemical Communications,2011,47,pp. 961-963

[16] Y. Hou, Q. Lu, J. Deng, H. Li, Y. Zhang, One-potelectrochemical synthesis offunctionalized fluorescent carbon dots and their selective sensing for mercury ion., Analytica Chimica Acta, 2015, 866, pp.69-74

[17] Liang Q., Ma W., Shi Y., Li Z., Yang X., Easy synthesis of highly fluorescent carbon quantum dots from gelatin and their luminescent properties and applications, Carbon, 2013, 60, pp. $421-428$ 